\title{
Nonlinear from linear states in two-component Bose-Einstein condensates
}

\author{
G Karali $^{1,2}$, P G Kevrekidis ${ }^{3}$ and N K Efremidis ${ }^{4}$ \\ ${ }^{1}$ Department of Applied Mathematics, University of Crete, GR 71409, Heraklion, Greece \\ 2 Institute for Applied and Computational Mathematics, FORTH, Crete, Greece \\ ${ }^{3}$ Department of Mathematics and Statistics, University of Massachusetts, Amherst, \\ MA 01003-4515, USA \\ ${ }^{4}$ Department of Applied Mathematics, University of Crete, GR 71409, Heraklion, Greece
}

Received 12 September 2008, in final form 13 November 2008

Published 19 December 2008

Online at stacks.iop.org/JPhysA/42/045206

\begin{abstract}
In this work, we consider the extension of linear eigenmodes of the harmonic oscillator potential into nonlinear states, for the case of twocomponent Gross-Pitaevskii equations with a parabolic potential, motivated by the context of two interacting hyperfine states of ${ }^{87} \mathrm{Rb}$ in Bose-Einstein condensates. In particular, we establish that nonlinear continuations of various eigenmode combinations are possible and corroborate this analytical finding with numerical computations for the lowest few eigenmode combinations involving the ground state and the first two excited states. A multitude of nonlinear states can be constructed in this way, some of which spontaneously deform, as the interactions become stronger, into previously obtained nonlinear eigenstates. The Bogolyubov-de Gennes analysis of the excitations on top of such states illustrates that some of them may become unstable beyond a critical threshold (of the chemical potentials associated with the states), while others may be stable within the entire range of chemical potentials considered herein. When the modes are found to be unstable, their evolution is followed, leading to interesting dynamical effects such as spontaneous symmetry breaking or oscillatory growth.
\end{abstract}

PACS numbers: 03.75.Lm, 03.75.Mn

(Some figures in this article are in colour only in the electronic version)

\section{Introduction}

The experimental realization of Bose-Einstein condensation (BEC) in the mid-1990s sparked an ever-expanding interest in the study of both nonlinear wave and fundamental quantum phenomena that arise in that setting $[1,2]$. The existence of a mean-field model that accurately 
describes the BEC's near-zero temperature was a source of an intense and wide range of investigations on coherent structures that emerge in such BECs, due to the effective nonlinearity induced by the inter-particle interaction. Such studies allowed us to monitor macroscopic nonlinear matter waves including bright matter-wave solitons in attractive BECs [3-5], as well as dark [6-9] and gap [10] matter-wave solitons in repulsive BECs (see also the recent reviews [11]).

One of the particularly interesting aspects of BECs that has been a focal point of many investigations is the statics and dynamics of binary mixtures [12-14]. The latter display rich phase separation phenomena due to the nonlinear interactions between the different atomic species/states. The formation of robust single- and multi-ring patterns [13, 15], the evolution of initially coincident triangular vortex lattices through a turbulent regime into an interlaced square vortex lattice state [16] in coupled hyperfine states of ${ }^{87} \mathrm{Rb}$, or the study in optical traps of different Zeeman levels of ${ }^{23} \mathrm{Na}$ forming striated magnetic domains $[17,18]$ are only a small sample among the many exciting possibilities that multi-component BECs can offer. Such investigations are still very active presently, with an interesting recent example being the examination of structural phase transitions from miscibility to immiscibility as a Feshbach resonance [19-21] is crossed [22].

In parallel to the experimental developments, a large volume of theoretical studies were devoted to an effort to understand the relevant phenomenology associated with phase separation. These studies demonstrated that the stationary state of a BEC mixture depends critically on the intra- and inter-species scattering lengths, as does its stability against excitations [23-26]. Additional aspects examined involved the static and dynamic properties [27-31], including the excitation spectrum and the nature of low-frequency simultaneous collective excitations, and solitary waves [32-36] (see also references therein).

Our purpose in the present paper is to revisit the topic of multi-component condensates but from a point of view that has been recently particularly fruitful in obtaining existence (and stability) results for single-component condensates, namely that of the underlying linear limit; for single-component results, see, e.g., the analysis of [37-42]. Here, we consider a two-component version of such considerations, whereby each of the components can be initialized in each of the first few linear states. The theoretical background of the existence analysis is given in section 2, based on a small amplitude expansion near the linear limit and deriving the corresponding persistence conditions (this is tantamount to a 'tight-binding' approximation). Then, in section 3, we illustrate the relevant modes numerically, identifying them in the vicinity of the linear limit and examining their linear stability (and dynamics, when they are found to be unstable), as their intensity is increased, through an increase in the corresponding chemical potential. Finally, in section 4, we summarize our findings and present our conclusions, as well as some possible directions for future study.

\section{Tight-binding approximation}

Consider the physically relevant system of two BECs as described in the mean-field approximation by two coupled Gross-Pitaevskii equations (GPEs),

$$
\begin{aligned}
& \mathrm{i} \hbar \psi_{1 T}+\frac{\hbar^{2}}{2 m} \psi_{1 \xi \xi}-\left(g_{11}\left|\psi_{1}\right|^{2}+g_{12}\left|\psi_{2}\right|^{2}\right) \psi_{1}-V(\xi) \psi_{1}=0 \\
& \mathrm{i} \hbar \psi_{2 T}+\frac{\hbar^{2}}{2 m} \psi_{2 \xi \xi}-\left(g_{12}\left|\psi_{1}\right|^{2}+g_{22}\left|\psi_{2}\right|^{2}\right) \psi_{2}-V(\xi) \psi_{2}=0,
\end{aligned}
$$

where $\psi_{1}, \psi_{2}$ are the two BEC components, $\tau$ and $\xi$ are the temporal and spatial coordinates, $\hbar$ is reduced Planck's constant, $g_{j, j}$ are the cubic nonlinear coefficients, $g_{j, k} j \neq k$ are the 
Table 1. Parameters of equation (2.3) some of the lower combinations $|0,0\rangle,|0,1\rangle,|1,1\rangle,|1,2\rangle$, $|2,2\rangle$. For explanation of the relevant notations, see the text.

\begin{tabular}{llllll}
\hline & $s_{11}$ & $s_{12}$ & $s_{21}$ & $s_{22}$ & $t / \tau$ \\
\hline$|0,0\rangle$ & $1.004 /(4 \sqrt{2 \pi})$ & $0.9766 /(4 \sqrt{2 \pi})$ & $0.9766 /(4 \sqrt{2 \pi})$ & $0.95 /(4 \sqrt{2 \pi})$ & $4 \sqrt{2 \pi}$ \\
$|0,1\rangle$ & $1.004 /(4 \sqrt{2 \pi})$ & $0.9766 /(2 \sqrt{2 \pi})$ & $0.9766 /(2 \sqrt{2 \pi})$ & $3 \times 0.95 /(8 \sqrt{\pi})$ & $4 \sqrt{2 \pi}$ \\
$|1,1\rangle$ & $3 \times 1.004 \sqrt{2} /(8 \sqrt{\pi})$ & $3 \times 0.9766 \sqrt{2} /(8 \sqrt{\pi})$ & $3 \times 0.9766 \sqrt{2} /(8 \sqrt{\pi})$ & $3 \times 0.95 \sqrt{2} /(8 \sqrt{\pi})$ & $8 \sqrt{\pi} /(3 \sqrt{2})$ \\
$|1,2\rangle$ & $3 \times 1.004 \sqrt{2} /(8 \sqrt{\pi})$ & $7 \times 0.9766 \sqrt{2} /(32 \sqrt{\pi})$ & $7 \times 0.9766 /(32 \sqrt{\pi})$ & $41 \times 0.95 /(32 \sqrt{\pi})$ & 1 \\
$|2,2\rangle$ & $1.004 / \sqrt{2 \pi}$ & $3 \times 0.9766 /(8 \sqrt{2 \pi})$ & $3 \times 0.9766 /(16 \sqrt{\pi})$ & $41 \times 0.95 /(32 \sqrt{\pi})$ & 1 \\
\hline
\end{tabular}

nonlinear couplings between the two components and $m$ is the mass for ${ }^{87} \mathrm{Rb}$ (which is the case that we focus on herein, although our considerations are fairly general). Setting $t=T / \hbar$ and $x=\xi /(\hbar / \sqrt{2 m})$ along with $g_{i j}=4 \pi \hbar^{2} a_{i j} / m, u=\left(100 a_{0} 4 \pi \hbar^{2} / m\right)^{1 / 2} \psi_{1}, v=$ $\left(100 a_{0} 4 \pi \hbar^{2} / m\right)^{1 / 2} \psi_{2}$ we obtain the system under discussion

$$
\begin{aligned}
& \mathrm{i} u_{t}+\frac{1}{2} u_{x x}-\left(\frac{a_{11}}{100 a_{0}}|u|^{2}+\frac{a_{12}}{100 a_{0}}|v|^{2}\right) u-V(x) u=0, \\
& \mathrm{i} v_{t}+\frac{1}{2} v_{x x}-\left(\frac{a_{12}}{100 a_{0}}|u|^{2}+\frac{a_{22}}{100 a_{0}}|v|^{2}\right) v-V(x) v=0 .
\end{aligned}
$$

Note that in the case of magnetic traps $V(x)=\Omega^{2} x^{2} / 2$. In the linear limit $\left(a_{i j}=0\right)$ both of the above equations reduce to the linear eigenvalue problem of the Schrödinger equation

$$
\mathrm{i} q_{t}+\frac{1}{2} q_{x x}+V(x) q=0
$$

that supports Hermite polynomial solutions of the form $q_{n}=\mathrm{e}^{-\mathrm{i} E_{n} t} f_{n}(x)$ where

$$
f_{n}(x)=2^{-\frac{n}{2}} \pi^{-1 / 4}(n !)^{-1 / 2} \mathrm{e}^{-\frac{\Omega x^{2}}{2}} H_{n}(\sqrt{\Omega} x),
$$

and $E_{n}=\Omega(n+1 / 2)$. In the nonlinear case, we apply a tight-binding approximation,

$$
u=\epsilon^{\frac{1}{2}} A(t \epsilon) q_{m}, \quad v=\epsilon^{\frac{1}{2}} B(t \epsilon) q_{n},
$$

where $A, B$ are the time-varying amplitudes of the two modes and $q_{m}, q_{n}$ are described above. The $O\left(\epsilon^{1 / 2}\right)$ terms are identically satisfied. The next order $O\left(\epsilon^{3 / 2}\right)$ terms satisfy

$$
\begin{aligned}
& \mathrm{i} A_{t} q_{m}-\frac{a_{11}}{100 a_{0}}|A|^{2} A q_{m}^{3}-\frac{a_{12}}{100 a_{0}} A|B|^{2} q_{n}^{2} q_{m} A=0, \\
& \mathrm{i} B_{t} q_{n}-\frac{a_{12}}{100 a_{0}}|A|^{2} B q_{m}^{2} q_{n}-\frac{a_{22}}{100 a_{0}} B|B|^{2} q_{n}^{3}=0 .
\end{aligned}
$$

We next project equations (2.2) on $q_{m}, q_{n}$, and set $s_{11}=\sigma\left(a_{11}\left\langle q_{m}^{3}, q_{m}\right\rangle\right) /\left(100 a_{0}\left\langle q_{m}, q_{m}\right\rangle\right)$, $s_{12}=\sigma\left(a_{12}\left\langle q_{n}^{2} q_{m}, q_{m}\right\rangle\right) /\left(100 a_{0}\left\langle q_{m}, q_{m}\right\rangle\right), s_{21}=\sigma\left(a_{12}\left\langle q_{m}^{2} q_{n}, q_{n}\right\rangle\right) /\left(100 a_{0}\left\langle q_{n}, q_{n}\right\rangle\right), s_{22}=$ $\sigma\left(a_{22}\left\langle q_{n}^{3}, q_{n}\right\rangle\right) /\left(100 a_{0}\left\langle q_{n}, q_{n}\right\rangle\right)$, where we use the braket notation $\langle f, g\rangle=\int_{-\infty}^{\infty} f^{*}(x) g(x) \mathrm{d} x$. The resulting system reads

$$
\mathrm{i} A_{\tau}-\left(s_{11}|A|^{2}+s_{12}|B|^{2}\right) A=0 \quad \mathrm{i} B_{\tau}-\left(s_{21}|A|^{2}+s_{22}|B|^{2}\right) B=0,
$$

where for ${ }^{87} \mathrm{Rb}$ atoms $a_{11}=100.40 a_{0}, a_{12}=97.66 a_{0}, a_{22}=95.00 a_{0}$ (see, e.g., [15]), and $t / \tau=\sigma$. In table 1 , the parameters for some of the prototypical combinations of the lowest condensate modes are depicted. In the table (and hereafter), we use the notation $|m, n\rangle$ for a state combining, in the linear limit, an eigenmode of order $m$ in the first component with an eigenmode of order $n$ in the second component. Stationary solutions of the previous system 
of equations are found by setting

$$
A=\alpha \mathrm{e}^{-\mathrm{i} \mu_{1} \tau}, \quad B=\beta \mathrm{e}^{-\mathrm{i} \mu_{2} \tau},
$$

where $\mu_{1}=s_{11} \alpha^{2}+s_{12} \beta^{2}$ and $\mu_{2}=s_{21} \alpha^{2}+s_{22} \beta^{2}$ are the chemical potentials. It is important to point out that the existence of solutions to this algebraic system of equations guarantees the presence of the corresponding state near the linear limit.

\section{Numerical results}

We now test the predictions of the previous section numerically, by identifying stationary states for $u(x)$ and $v(x)$, the two BEC components, through fixed-point iterations of equation (2.1). For definiteness, these iterations have been performed with the values of the scattering lengths $a_{11}=100.4 a_{0}, a_{22}=95 a_{0}$ and $a_{12}=150 a_{0}$. The first two are identical to the values used, e.g., in the recent experimental and numerical investigation of [15] (and also in the theoretical analysis above). The third one is assumed to be tuned by employing either magnetic [19, 20] or optical Feshbach resonances [21]. The latter permits the flexibility of tuning the s-wave scattering length of a particular type of interatomic interactions. This particular set of $a_{i j}$ 's is placed well within the immiscible regime and permits us to compare our obtained solutions with other phase-separated types of solutions which have been proposed within this regime; see, e.g., [32] where a similar choice of $a_{i j}$ 's was used. In this way, the relevant and interesting phase separation dynamical phenomena are more pronounced and clearly observable in what follows.

In addition to the existence of the relevant nonlinear states, we perform a linear stability analysis (a Bogolyubov-de Gennes analysis) to identify whether the obtained waveforms are stable or unstable. This is done through the use of an ansatz,

$$
\begin{aligned}
& \psi_{1}=\exp \left(-\mathrm{i} \mu_{1} t\right)\left[u(x)+\delta\left(U_{1}(x) \exp (\lambda t)+U_{2}(x) \exp \left(\lambda^{\star} t\right)\right)\right] \\
& \psi_{2}=\exp \left(-\mathrm{i} \mu_{2} t\right)\left[v(x)+\delta\left(V_{1}(x) \exp (\lambda t)+V_{2}(x) \exp \left(\lambda^{\star} t\right)\right)\right]
\end{aligned}
$$

and the subsequent solution of the ensuing linear eigenvalue problem at $O(\delta)$ for $\lambda$ and the corresponding eigenvector $\left[U_{1}, U_{2}^{\star}, V_{1}, V_{2}^{\star}\right]^{T} ; \delta$ here is a formal small parameter. The existence of eigenvalues $\lambda$ with nonzero real part corresponds to instability (given the symplectic Hamiltonian nature of the eigenvalue problem, which necessitates that if $\lambda$ is an eigenvalue, then so are $-\lambda, \lambda^{\star}$ and $\left.-\lambda^{\star}\right)$.

Figure 1 shows the case of the $|0,0\rangle$ state, illustrated through continuations as a function of the chemical potential $\mu_{1} ; \mu_{2}=\mu_{1}+0.022$ is used in this set of results. This is a choice which is representative of the results that can be obtained in the larger two-parameter space of $\left(\mu_{1}, \mu_{2}\right)$; such typical choices (of mono-parametric continuations) have been made in all the cases shown in what follows of the solution branches of the two components. We can see that the $|0,0\rangle$ branch is, in fact, stable sufficiently close to the linear limit of the magnetic trap of $\Omega=0.1$ and it becomes unstable due to a real eigenvalue pair for $\mu_{1}>0.22$. However, it should be noted that as the chemical potential is raised to large values, this instability becomes weaker (its maximum growth rate occurs at $\mu_{1}=0.34$ ), until effectively the configuration can be considered as stable for values of $\mu_{1}>1$. It is interesting to note also that for sufficiently large values of the chemical potential, the repulsive interaction between the components enforces a phase separation, which systematically modifies this state toward the type of waveform previously observed, e.g., in figure 2 (top panel) of [32] (note that this eigenfunction for lower values of the chemical potential also relates to waveforms of figure 1 (bottom panel) of [32]; our continuation illustrates how these two profiles may 

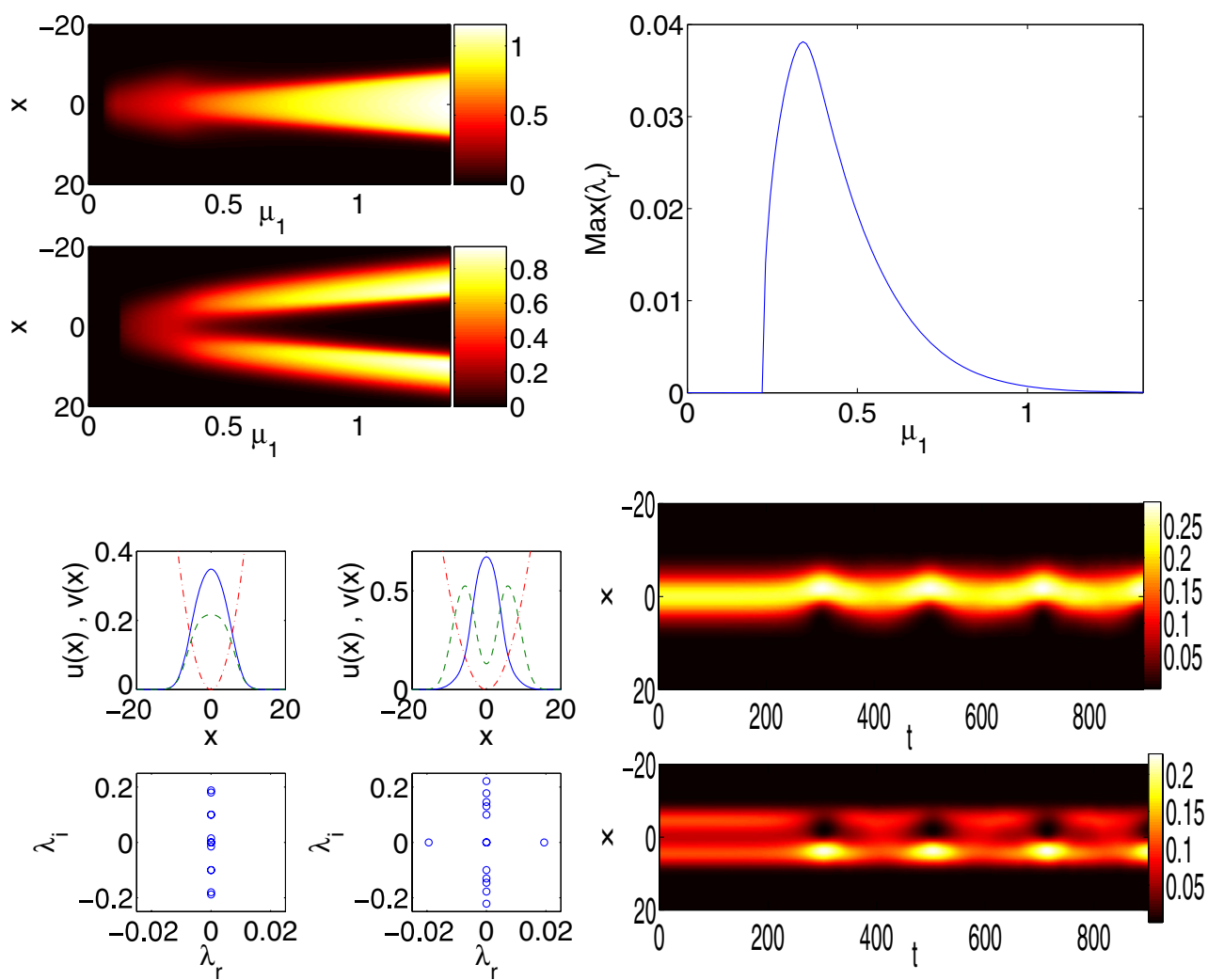

Figure 1. The two top left panels show a continuation of the two components $(u(x)$ and $v(x))$ of the solution as a function of the chemical potential $\mu_{1}$. The top right panel shows the most unstable eigenvalue of the configuration. Note that the instability of this $|0,0\rangle$ mode disappears for $\mu_{1} \leqslant 0.22$ and becomes also very weak for sufficiently large $\mu_{1}$. The bottom left four panels show the profiles (first row) and the corresponding stability (second row) of such a mode for $\mu_{1}=0.2$ (left panels) and for $\mu_{1}=0.5$ (right panels). The profile of the first component is shown by solid line, and of the second by dashed line, while the parabolic trap is shown by a dash-dotted line. The right panel shows the dynamical evolution of the instability for $\mu_{1}=0.35$ in the form of a spacetime contour plot of the density of the respective components.

correspond to different limits of the same branch). Furthermore, we have examined the dynamics of this branch, an example of which is shown in the bottom right panel of figure 1 . What can be seen is that the result of the instability is to induce a spontaneous symmetry breaking in the dynamics of the multi-component condensate around $t \approx 300$. This, in turn, seems to suggest that such destabilization appears to favor the spontaneous formation of stable domain wall type states such as those considered in the bottom panel of figure 2 in [32].

However, a different type of waveform, which, in fact, turns out to be linearly stable (in the parameter range considered herein) is given by the $|0,1\rangle$ branch of figure 2 . In this case also, we can observe that the two lobes of the anti-symmetric wavefunction are pushed away from the center, as the chemical potential increases, as a result of the repulsive inter-species interaction and concomittant immiscibility between the components. In the continuations shown in figure $2, \mu_{2}=\mu_{1}+0.064$ was used.

On the other hand, the $|1,1\rangle$ branch turns out to have a somewhat similar stability picture to that of the $|0,0\rangle$ solution. In particular, while for small values of $\mu_{1}$, the branch is stable 

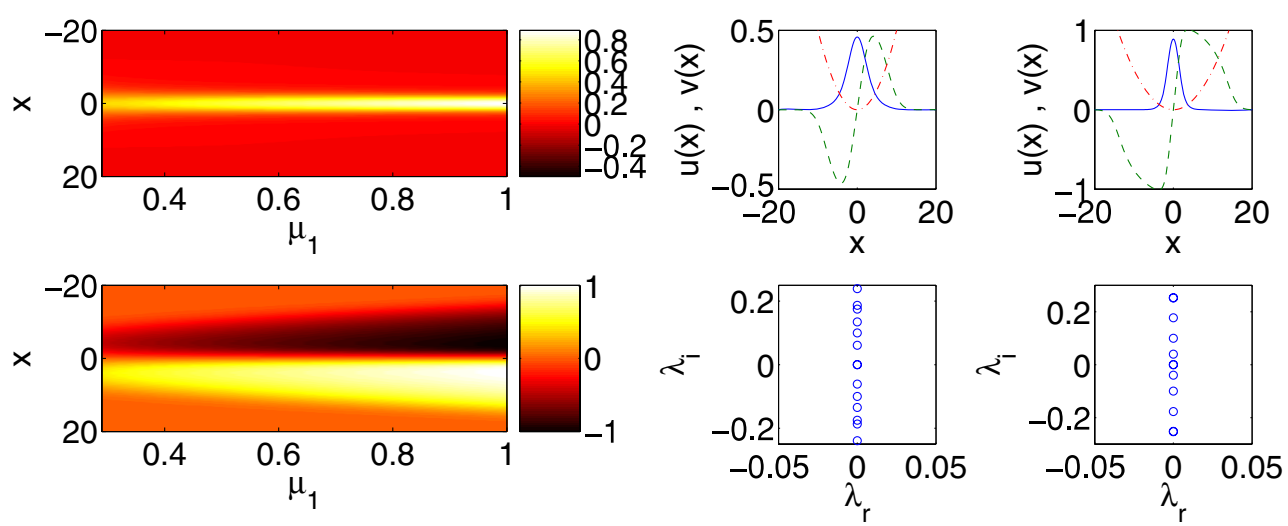

Figure 2. Same as in figure 1, but now for the $|0,1\rangle$ mode. Since this waveform is always found to be stable, in the right panel we do not show its largest real eigenvalue, but rather the mode (top) and its stability (bottom) for $\mu_{1}=0.3$ (second column) and $\mu_{1}=1$ (third column).

sufficiently close to the linear limit, as $\mu_{1}$ increases past the value of $\mu_{1}>0.31$, the solution becomes unstable due to a real eigenvalue pair which becomes maximal for $\mu_{1}=0.48$ and then decreases for larger values of the chemical potential; $\mu_{2}=\mu_{1}-0.01$ was used here. As the chemical potential increases, the lobes of the wavefunctions of the two components once again grow further apart, showcasing the phase separation between the two states. Another element of similarity between this branch and the $|0,0\rangle$ case can be found in connection to their dynamical evolution. It can be seen from the typical time-stepping example shown in the bottom right panel of figure 3 for the case of $\mu_{1}=0.5$ that once again a spontaneous symmetry breaking emerges for times $t \approx 200$, although the dark soliton-type structure centered at $x=0$ in each of the components appears to persist for considerably longer times. Similarly to what was seen in the case of $|0,0\rangle$, the symmetry breaking appears to have a recurrent emergence in the dynamics, presumably due to the Hamiltonian nature of the model.

We also examined the mixed modes involving the second excited state. As such an example, in figure 4 , we illustrate the $|0,2\rangle$ mode, initialized for $\mu_{1}$ close to the linear limit (and $\mu_{2}=\mu_{1}+0.2$ ) and continued over $\mu_{1}$ up to values of $O(1)$. In this case also, we find, similarly to the setting of $|0,1\rangle$ that the branch is stable for all values of the parameters considered herein, as is also shown in the case example of the right panels of the figure. It is interesting to note here that the strong cross-repulsion between the components, in combination with the peak of the $|2\rangle$ branch at $x=0$ lead to the splitting of the $|0\rangle$ branch into a two-hump structure, similarly to what was observed in the $|0,0\rangle$ case.

Finally, we also show the $|1,2\rangle$ branch in figure 5. As can be seen in the top row's right panel of the figure (as well as at the case examples of the bottom row), this branch may, in fact, be unstable for the parameter range examined due to an oscillatory instability. In the case examined, where $\mu_{2}=\mu_{1}+0.1$, the instability exists for $\mu_{1}<0.47$ and for $1.1<\mu_{1}<1.16$. In this case, the example of instability demonstrated in the bottom panel of figure 5 is due to a complex eigenvalue quartet with a very weak growth rate (i.e., with a very small real part). For this reason, the eventual manifestation of the instability arises for much longer times than in the earlier examples, i.e., for $t>1500$, for the value of $\mu_{1}=0.4$ shown in the figure. Interestingly, the oscillatory growth of the two components leads to a destruction of the waveform of the first component and to apparently irregular dynamics. It should be noted here that the second instability window mentioned above is of similar type as the first one. In 

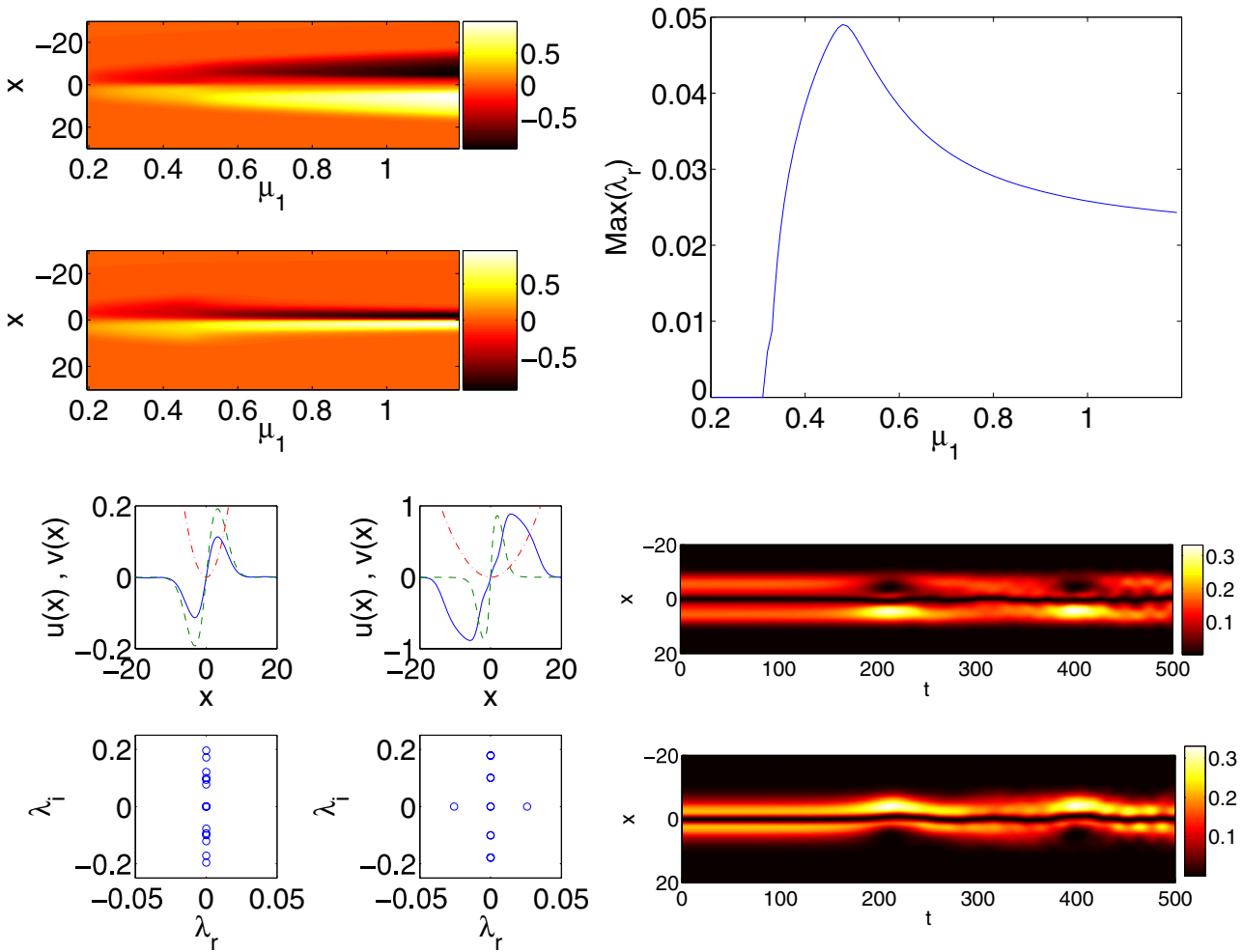

Figure 3. Same as in the case of figure 1, but now for the $|1,1\rangle$ mode. The bottom left two rows show the mode and its stability for $\mu_{1}=0.2$ (left panels) and for $\mu_{1}=1$ (right panels), while the bottom right panels show the dynamical evolution of the two components for this mode with $\mu_{1}=0.5$.
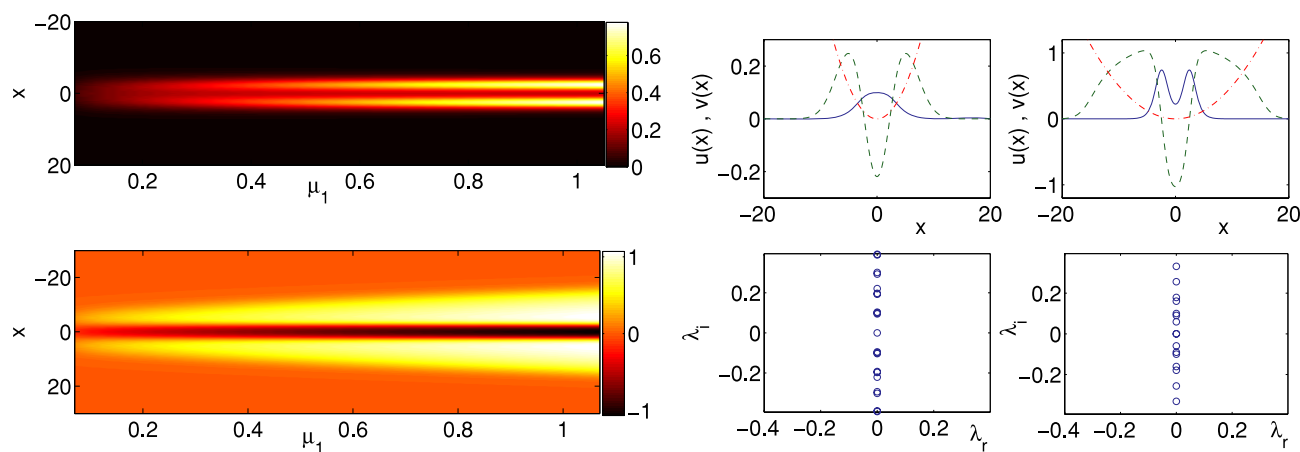

Figure 4. Same as in the case of figure 2, but now for the $|0,2\rangle$ branch. It is interesting to note that in this case the branch is found to be linearly stable analogously to what we found for the $|0,1\rangle$ case. The right panels are for $\mu_{1}=0.1$ and $\mu_{1}=1$ respectively; $\mu_{2}=\mu_{1}+0.2$ here.

particular, as $\mu$ is increased, the two pairs whose resonance gave rise to the first instability window no longer resonate for $\mu>0.47$. Further increase of the chemical potential leads one of these pairs to move along the imaginary axis of eigenvalues and eventually collide with 

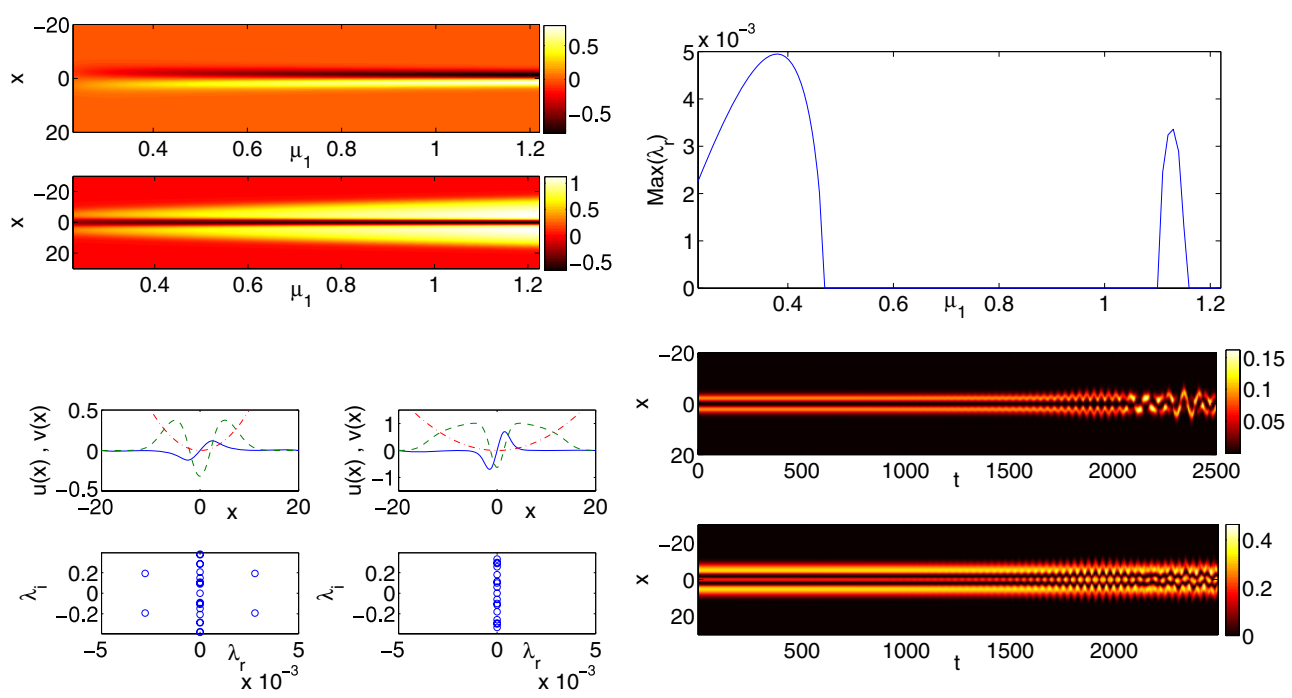

Figure 5. Same as in the case of figure 1, but now for the $|1,2\rangle$ branch. The right panel shows the maximal growth rate of the instability revealing the potential presence of oscillatory instabilities. The bottom left panel profiles and their corresponding spectral planes are shown for $\mu_{1}=0.25$ and $\mu_{1}=1 ; \mu_{2}=\mu_{1}+0.1$. The bottom right panel shows the dynamical evolution for the case of $\mu_{1}=0.4$.

another pair of opposite signature (which was originally at a higher frequency), thus giving rise to the second instability window.

\section{Conclusions}

In this paper, we showed that two-component condensates have a wealth of states that have not been previously explored (or have only partially been explored in the strongly nonlinear limit, e.g., in [32]). Such states can be generated from the underlying linear limit of the system with the parabolic potential through a combination of different eigenfunctions (indexed by the order of the corresponding Hermite mode) in each component. The existence of such states can be established in the vicinity of the linear limit, through a small amplitude expansion and demonstration of the solvability of the ensuing persistence conditions. The waveforms have also been identified numerically and their linear stability has been explored. It has been observed that they may undergo instabilities (through the bifurcation of "symmetry broken', domain-wall-type states), or in fact, as is the case especially for mixed modes, combining different linear eigenstates, may be stable in wide parametric intervals. This suggests the potential experimental realizability of such states. In fact, for some of these coherent structures, one may argue that they, or higher dimensional analogs thereof, have already been experimentally observed. For instance, the $|0,1\rangle$ state is tantamount to the bright-dark soliton that was recently experimentally identified in the work of [36]. In that experiment, a phase mask was applied to an elongated ${ }^{87} \mathrm{Rb} \mathrm{BEC}$ in the $F=1$ hyperfine state, to produce a dark soliton. At the same time, a fraction of atoms within the phase mask were transferred to the $F=2$ hyperfine state, creating a bright soliton therein, thus giving rise to the first experimental realization in the two-component system of a bright-dark soliton. 
It is important here to highlight the physical context in which our findings above may be relevant. To that effect, we use the theoretical work of [43], setting the stage for the validity of the reduction of the BEC dynamics to a quasi-1D setting, as well as the very recent experimental work of [44]. A key diagram to use in this regard is figure 1 of [43]. According to that, the ideal gas regime (which is tantamount to our linear regime) is relevant for $\alpha_{\perp} / \alpha>10^{2}$; here $\alpha_{\perp}=\sqrt{\hbar /\left(m \omega_{\perp}\right)}$, where $\omega_{\perp}$ is the transverse trapping frequency of the cloud and $a$ is a typical value of the scattering length (say, $a_{11}$ for our purposes). As one increases the number of atoms (for a fixed $\Omega$ ) one passes from the ideal gas regime to the one-dimensional (1D) mean-field regime (when crossing an appropriate curve). In our case we used a typical $\Omega=0.1$. Assuming a transverse trapping frequency of about $2 \pi \times 100 \mathrm{~Hz}$ (which is fairly reasonable in experimental settings such as that of [44]), we obtain that $\alpha_{\perp} / \alpha \approx 193>10^{2}$. Then as the number of atoms increases, we start from the linear state and can get to numbers of atoms of up to a few hundred (which is feasible in the experiment of [44]) and in fact up to 1000-1500. For $N=1000, N \Omega=100$, which according to figure 1 of [43] is still within the 1D mean-field regime. In fact, this is precisely the point of our numerical simulations, as well. We do not mean to imply that our linear states will be observable since states with that few atoms may indeed be hard to monitor; what we argue instead is that, within the context of the two-component GPE model with the parabolic potential, these are legitimate starting points for identifying states that will survive in the 1D mean-field limit (that can realistically be described by the partial differential equations used here) even for numbers of atoms which are relevant to physical experiments such as those reported in [44].

These results suggest a number of potential future directions. On the one hand, it would be particularly interesting to quantify theoretically both the number of unstable eigendirections of the multi-component problem, similarly, e.g. to the corresponding single-component analysis of [39]. Another direction of interest would be to generalize the present results to the higher dimensional case; while quasi-one-dimensional experimental settings for multi-component mixtures are presently being studied [36], most of the earlier experiments have been realized in the three-dimensional context (see, e.g., [15], and references therein). Finally, yet another vein of recent studies concerns the study of spinor condensates $[45,46]$ where the corresponding mean-field model consists of a three-component system in the $F=1$ case (see, e.g., [47], and references therein). An even more complex possibility consists of the five-component setting of the $F=2$ spinor condensate [48]. Extending the present considerations in such settings would be a challenging, yet relevant task.

\section{Acknowledgments}

GK is supported by a Marie Curie International Reintegration Grant within the 7th European Community Framework Programme, MIRG-CT-2007-200526 and a research grant at the University of Crete, ELKE 2740. PGK gratefully acknowledges support from NSF-CAREER, NSF-DMS-0505663, NSF-DMS-0619492, NSF-DMS-0806762, as well as from a Research Fellowship of the Alexander von Humboldt Foundation.

\section{References}

[1] Pethick C J and Smith H 2002 Bose-Einstein Condensation in Dilute Gases (Cambridge: Cambridge University Press)

[2] Pitaevskii L P and Stringari S 2003 Bose-Einstein Condensation (Oxford: Oxford University Press)

[3] Strecker K E, Partridge G B, Truscott A G and Hulet R G 2002 Nature 417150 
[4] Khaykovich L, Schreck F, Ferrari G, Bourdel T, Cubizolles J, Carr L D, Castin Y and Salomon C 2002 Science 2961290

[5] Cornish S L, Thompson S T and Wieman C E 2006 Phys. Rev. Lett. 96170401

[6] Burger S, Bongs K, Dettmer S, Ertmer W, Sengstock K, Sanpera A, Shlyapnikov G V and Lewenstein M 1999 Phys. Rev. Lett. 835198

[7] Denschlag J et al 2000 Science 28797

[8] Anderson B P, Haljan P C, Regal C A, Feder D L, Collins L A, Clark C W and Cornell E A 2001 Phys. Rev. Lett. 862926

[9] Dutton Z, Budde M, Slowe Ch and Hau L V 2001 Science 293663

[10] Eiermann B, Anker Th, Albiez M, Taglieber M, Treutlein P, Marzlin K-P and Oberthaler M K 2004 Phys. Rev. Lett. 92230401

[11] Kevrekidis P G, Frantzeskakis D J and Carretero-González R (ed) 2008 Emergent Nonlinear Phenomena in Bose-Einstein Condensates. Theory and Experiment (Berlin: Springer)

See also Carretero-González R, Kevrekidis P G and Frantzeskakis D J 2008 Nonlinearity 21 R139

[12] Myatt C J, Burt E A, Ghrist R W, Cornell E A and Wieman C E 1997 Phys. Rev. Lett. 78586

[13] Hall D S, Matthews M R, Ensher J R, Wieman C E and Cornell E A 1998 Phys. Rev. Lett. 811539

[14] Stamper-Kurn D M, Andreas M R, Chikkatur A P, Inouye S, Miesner H-J, Stenger J and Ketterle W 1998 Phys. Rev. Lett. 802027

[15] Mertes K M, Merrill J W, Carretero-González R, Frantzeskakis D J, Kevrekidis P G and Hall D S 2007 Phys. Rev. Lett. 99190402

[16] Schweikhard V, Coddington I, Engels P, Tung S and Cornell E A 2004 Phys. Rev. Lett. 93210403

[17] Miesner H-J, Stamper-Kurn D M, Stenger J, Inouye S, Chikkatur A P and Ketterle W 1999 Phys. Rev. Lett. 822228

[18] Stenger J, Inouye S, Stamper-Kurn D M, Miesner H-J, Chikkatur A P and Ketterle W 1999 Nature 396345

[19] Köhler T, Goral K and Julienne P S 2006 Rev. Mod. Phys. 781311

[20] Inouye S, Andrews M R, Stenger J, Miesner H J, Stamper-Kurn D M and Ketterle W 1998 Nature 392151

Stenger J, Inouye S, Andrews M R, Miesner H-J, Stamper-Kurn D M and Ketterle W 1999 Phys. Rev. Lett. 822422

Roberts J L, Claussen N R, Burke J P Jr, Greene C H, Cornell E A and Wieman C E 1998 Phys. Rev. Lett. 815109

Cornish S L, Claussen N R, Roberts J L, Cornell E A and Wieman C E 2000 Phys. Rev. Lett. 851795

[21] Fatemi F K, Jones K M and Lett P D 2000 Phys. Rev. Lett. 854462

Theis M, Thalhammer G, Winkler K, Hellwig M, Ruff G, Grimm R and Denschlag J H 2004 Phys. Rev. Lett. 93123001

[22] Papp S B, Pino J M and Wieman C E 2008 arXiv:0802.2591

[23] T-L Ho and Shenoy V B 1996 Phys. Rev. Lett. 773276

[24] Esry B D, Greene C H, Burke J P and Bohn J L 1997 Phys. Rev. Lett. 783594

[25] Law C K, Pu H, Bigelow N P and Eberly J H 1997 Phys. Rev. Lett. 793105

[26] Ao P and Chui S T 1998 Phys. Rev. A 584836

[27] Goldstein E V and Meystre P 1997 Phys. Rev. A 552935

[28] Busch T, Cirac J I, Pérez-García V M and Zoller P 1997 Phys. Rev. A 562978

[29] Graham R and Walls D 1998 Phys. Rev. A 57484

[30] Esry B D and Greene C H 1998 Phys. Rev. A 571265

[31] Pu H and Bigelow N P 1998 Phys. Rev. Lett. 801134

[32] Trippenbach M, Góral K, Rzazewski K, Malomed B and Band Y B 2000 J. Phys. B: At. Mol. Opt. Phys. 334017

[33] Kevrekidis P G, Nistazakis H E, Frantzeskakis D J, Malomed B A and Carretero-González R 2004 Eur. Phys. J. D 28181

[34] Cruz H A, Brazhnyi V A, Konotop V V, Alfimov G L and Salerno M 2007 Phys. Rev. A 76013603

[35] Pérez-García V M and Belmonte-Beitia J 2005 Phys. Rev. A 72033620

[36] Becker C, Stellmer S, Dörscher P Soltan-Panahi S, Baumert M, Richter E-M, Kronjäger J, Bongs K and Sengstock K 2008 Natl. Phys. 4496

[37] Kivshar Yu S, Alexander T J and Turitsyn S K 2001 Phys. Lett. A 278225

[38] Kevrekidis P G, Konotop V V, Rodrigues A and Frantzeskakis D J 2005 J. Phys. B: At. Mol. Opt. Phys. 381173

[39] Kapitula T and Kevrekidis P G 2005 Chaos 15037114

[40] Kapitula T, Kevrekidis P G and Frantzeskakis D J 2008 Chaos 18023101

[41] Alfimov G L and Zezyulin D A 2007 Nonlinearity 202075

[42] Zezyulin D A, Alfimov G L, Konotop V V and Pérez-García V M 2008 Phys. Rev. A 78013606

[43] Menotti C and Stringari S 2002 Phys. Rev. A 66043610 
[44] Weller A, Ronzheimer J P, Gross C, Frantzeskakis D J, Theocharis G, Kevrekidis P G, Esteve J and Oberthaler M K 2008 arXiv:0803.4352

[45] Stamper-Kurn D M, Andrews M R, Chikkatur A P, Inouye S, Miesner H-J, Stenger J and Ketterle W 1998 Phys. Rev. Lett. 802027

[46] Chang M-S, Hamley C D, Barrett M D, Sauer J A, Fortier K M, Zhang W, You L and Chapman M S 2004 Phys. Rev. Lett. 92140403

[47] Dabrowska-Wüster B J, Ostrovskaya E A, Alexander T J and Kivshar Yu S 2007 Phys. Rev. A 75032617

[48] Saito H and Ueda M 2005 Phys. Rev. A 72053628 